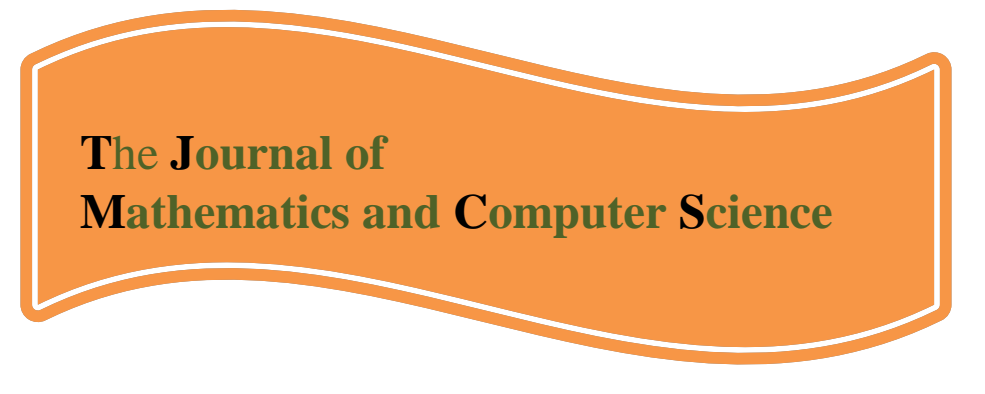

Available online at

\title{
http://www.TIMCS.com
}

\section{Solving Nonlinear Fractional Differential Equations by Bernstein Polynomials Operational Matrices}

\author{
Mohsen Alipour \\ Department of Mathematics, Imam Khomeini International University, \\ P.O. Box 34149-16818, Qazvin, Iran \\ m.alipour2323@gmail .com \\ Davood Rostamy \\ Department of Mathematics, Imam Khomeini International University, \\ P.0. Box 34149-16818, Qazvin, Iran \\ rostamy@khayam.ut.ac.ir
}

Received: February 2012, Revised: November 2012

Online Publication: December 2012

\begin{abstract}
In this paper, we solve nonlinear fractional differential equations by Bernstein polynomials. Firstly, we derive the Bernstein polynomials (BPs) operational matrix for the fractional derivative in the Caputo sense, which has not been undertaken before. This method reduces the problems to a system of algebraic equations. The results obtained are in good agreement with the analytical solutions and the numerical solutions in open literatures. Also, the solutions approach to classical solutions as the order of the fractional derivatives approach to 1 .
\end{abstract}

Keywords: Nonlinear fractional differential equations, Bernstein polynomials, operational matrix, Caputo derivative

\section{Mathematics Subject Classification: Primary 34A08; Secondary 76M22.}

\section{Introduction}

Differential equations of fractional order have been the focus of many studies due to their frequent appearance in various applications in fluid mechanics, viscoelasticity, biology, physics and engineering. Recently, a large amount of literatures developed concerning the application of fractional differential equations in nonlinear dynamics [1-4]. Several methods have been suggested to solve fractional differential equations, for example, homotopy methods [5-8], Adomian's decomposition method [9-11], variation iteration method [12, 13] and differential transform method [14]. 
In this work, we consider the nonlinear fractional differential equations as follows:

$D^{\alpha} y(t)=g(t, y(t)), \quad 0<t \leq 1$,

and the initial condition

$y^{(i)}(0)=y_{0}^{i}, \quad i=0,1, \ldots,\lceil\alpha\rceil-1$,

where $\alpha$ is constant and $g:[0,1] \times R \rightarrow R$ is polynomial function.

The rest of this paper is as follows. In Section 2, we present some preliminaries in fractional calculus. In Section 3, BPs are introduced and then we approximate functions by using BPs and we show the properties of BPs by several Lemmas and corollaries. We make a new operational matrix for fractional derivative by BPs in Section 4. In Section 5, we apply BPs for solving nonlinear fractional differential equations. In Section 6, numerical examples are simulated to demonstrate the high performance of the proposed method. Finally, Section 7 concludes our work in this paper.

\section{Some preliminaries in fractional calculus}

In this section, we give some basic definitions and properties of the fractional calculus which are used further in this paper.

Definition 2.1. (See [15]) We define

$C_{\mu}=\left\{f(t) \mid f(t)>0\right.$ for $t>0$ and $f(t)=t^{p} f_{1}(t)$ where $p>\mu$ and $\left.f_{1}(t) \in C[0, \infty)\right\}$, and $C_{\mu}^{n}=\left\{f(t) \mid f^{(n)}(t) \in C_{\mu}\right\}$ where $n \in N, \mu \in R$.

Definition 2.2. (See [15]) The Riemann-Liouville fractional integral operator of order $\alpha \geq 0$, of a function $f \in C_{\mu}, \mu \geq-1$, is defined as

$I^{\alpha} f(t)=\frac{1}{\Gamma(\alpha)} \int_{0}^{t}(t-x)^{\alpha-1} f(x) d x, \quad \alpha>0, t>0$,

$I^{0} f(t)=f(t)$,

and for $n-1<\alpha \leq n, n \in \mathrm{N}, t>0, f \in C_{-1}^{n}$, the fractional derivative of $f(t)$ in the Caputo sense is defined as

$D^{\alpha} f(t)=I^{n-\alpha} D^{n} f(t)=\frac{1}{\Gamma(n-\alpha)} \int_{0}^{t}(t-x)^{n-\alpha-1} f^{(n)}(x) d x$.

Property 2.3. (See [16-18]) For $f \in C_{\mu}, \mu \geq-1, \alpha, \beta \geq 0$ we have

$I^{\alpha} t^{\gamma}=\frac{\Gamma(\gamma+1)}{\Gamma(\alpha+\gamma+1)} t^{\alpha+\gamma}$

and for $n-1<\alpha \leq n, n \in \mathrm{N}$ and $f \in C_{\mu}^{n}, \mu \geq-1$ we see the following properties

1. $D^{\alpha} I^{\alpha} f(t)=f(t)$,

2. $I^{\alpha} D^{\alpha} f(t)=f(t)-\sum_{k=0}^{n-1} f^{(k)}\left(0^{+}\right) \frac{x^{k}}{k !}, t>0$,

3. $D^{\beta} f(t)=I^{\alpha-\beta} D^{\alpha} f(t)$. 


\section{Bernstein polynomials and their properties}

The Bernstein polynomials (BPs) of mth-degree are defined on the interval $[0,1]$ as follows:

$B_{i, m}(x)=\left(\begin{array}{l}m \\ i\end{array}\right) x^{i}(1-x)^{m-i}, \quad i=0,1, \cdots, m$

Corollary 3.1. Set $\left\{B_{0, m}(x), B_{1, m}(x), \cdots, B_{m, m}(x)\right\}$ is a complete basis in Hilbert space $L^{2}[0,1]$ and polynomials of degree $m$ can be expanded in terms of linear combination of $B_{i, m}(x)(i=0,1, \cdots, m)$ as follows

$P(x)=\sum_{i=0}^{m} c_{i} B_{i, m}(x)$.

Lemma 3.2. We can write $\Phi_{m}(x)=A T_{m}(x)$, where $A$ is a matrix upper triangular, $T_{m}(x)=\left[1, x, \ldots, x^{m}\right]^{T}$ and $\Phi_{m}(x)=\left[B_{0}(x), B_{2}(x), \ldots, B_{m}(x)\right]^{T}$.

Proof. Using binomial expansion of $(1-x)^{m-i}$, we have

$$
\begin{aligned}
B_{i, m}(x) & =\left(\begin{array}{l}
m \\
i
\end{array}\right) x^{i}(1-x)^{m-i}=\left(\begin{array}{l}
m \\
i
\end{array}\right) x^{i}\left(\sum_{k=0}^{m-i}(-1)^{k}\left(\begin{array}{c}
m-i \\
k
\end{array}\right) x^{k}\right) \\
& =\sum_{k=0}^{m-i}(-1)^{k}\left(\begin{array}{l}
m \\
i
\end{array}\right)\left(\begin{array}{c}
m-i \\
k
\end{array}\right) x^{i+k}, i=0,1 \ldots, m .
\end{aligned}
$$

Therefore we can write

$\Phi_{m}(x)=A T_{m}(x)$

where $A=\left(a_{i, j}\right)_{i, j=1}^{m+1}$ and $a_{i+1, j+1}=\left\{\begin{array}{ll}(-1)^{j-i}\left(\begin{array}{l}m \\ i\end{array}\right)\left(\begin{array}{c}m-i \\ j-i\end{array}\right) & i \leq j, \\ 0 & i>j,\end{array} \quad i, j=0,1, \ldots, m\right.$.

Lemma 3.3. (See [19]) Let $L^{2}[0,1]$ be a Hilbert space with the inner product $<f, g>=\int_{0}^{1} f(x) g(x) d x$, and $y \in L^{2}[0,1]$. Then, we can find the unique vector $c=\left[c_{1}, c_{2}, \cdots, c_{m}\right]^{T}$ such that

$y(x) \approx \sum_{i=0}^{m} c_{i} B_{i, m}(x)=c^{T} \Phi_{m}(x)$.

Corollary 3.4. In lemma 3.3, we have $c^{T}=<f, \Phi_{m}>Q^{-1}$, such that $<f, \Phi_{m}>=\int_{0}^{1} f(x) \Phi_{m}(x)^{T} d x=\left[<f, B_{0, m}>,<f, B_{1, m}>, \cdots,<f, B_{m, m}>\right]$ and $Q=\left(Q_{i, j}\right)_{i, j=1}^{m+1}$ is as follows 
$Q_{i+1, j+1}=\int_{0}^{1} B_{i, m}(x) B_{j, m}(x) d x=\frac{\left(\begin{array}{l}m \\ i\end{array}\right)\left(\begin{array}{l}m \\ j\end{array}\right)}{(2 m+1)\left(\begin{array}{l}2 m \\ i+j\end{array}\right)}, \quad i, j=0,1, \cdots m$.

Lemma 3.5. Suppose that $c_{(m+1) \times 1}$ is an arbitrary vector. The operational matrix of product $\hat{C}_{(m+1) \times(m+1)}$ using BPs can be given as follows:

$c^{T} \Phi_{m}(x) \Phi_{m}(x)^{T} \approx \Phi_{m}(x)^{T} \hat{C}$.

Proof. From (11) we have

$$
\begin{aligned}
c^{T} \Phi_{m}(x) \Phi_{m}(x)^{T} & =c^{T} \Phi_{m}(x)\left(T_{m}(x)^{T} A^{T}\right)=\left[c^{T} \Phi_{m}(x), x\left(c^{T} \Phi_{m}(x)\right), \ldots, x^{m}\left(c^{T} \Phi_{m}(x)\right)\right] A^{T} \\
& =\left[\sum_{i=0}^{m} c_{i} B_{i, m}(x), \sum_{i=0}^{m} c_{i} x B_{i, m}(x), \ldots, \sum_{i=0}^{m} c_{i} x^{m} B_{i, m}(x)\right] A^{T} .
\end{aligned}
$$

Now, we approximate all functions $x^{k} B_{i, m}(x)$ in terms of $\Phi_{m}(x)$. Thus we define $e_{k, i}=\left[e_{k, i}^{0}, e_{k, i}^{1}, \ldots, e_{k, i}^{m}\right]^{T}$, then by (12) we can write $x^{k} B_{i, m}(x) \approx e_{k, i} \Phi_{m}(x), \quad i, k=0,1, \ldots, m$.

So, we get

$$
\begin{aligned}
e_{k, i} & =Q^{-1}\left(\int_{0}^{1}\left(x^{k} B_{i, m}(x)\right) \Phi_{m}(x) d x\right) \\
& =Q^{-1}\left[\int_{0}^{1} x^{k} B_{i, m}(x) B_{0, m}(x) d x, \int_{0}^{1} x^{k} B_{i, m}(x) B_{1, m}(x) d x, \ldots, \int_{0}^{1} x^{k} B_{i, m}(x) B_{m, m}(x) d x\right]^{T} \\
& =\frac{Q^{-1}\left(\begin{array}{l}
m \\
i
\end{array}\right)}{2 m+k+1}\left[\frac{\left(\begin{array}{l}
m \\
0
\end{array}\right)}{\left(\begin{array}{l}
2 m+k \\
i+k
\end{array}\right)}, \frac{\left(\begin{array}{l}
m \\
1
\end{array}\right)}{\left(\begin{array}{l}
2 m+k \\
i+k+1
\end{array}\right)}, \ldots, \frac{\left(\begin{array}{l}
m \\
m
\end{array}\right)}{\left(\begin{array}{l}
2 m+k \\
i+k+m
\end{array}\right)}\right]^{T}, \quad i, k=0,1, \ldots, m .
\end{aligned}
$$

Then, we have

$$
\begin{aligned}
\sum_{i=0}^{m} c_{i} x^{k} B_{i, m}(x) & \approx \sum_{i=0}^{m} c_{i}\left(\sum_{j=0}^{m} e_{k, i}^{j} B_{j, m}(x)\right)=\sum_{i=0}^{m} B_{j, m}(x)\left(\sum_{i=0}^{m} c_{i} e_{k, i}^{j}\right) \\
& =\Phi_{m}(x)^{T}\left[\sum_{i=0}^{m} c_{i} e_{k, i}^{0}, \sum_{i=0}^{m} c_{i} e_{k, i}^{1}, \ldots, \sum_{i=0}^{m} c_{i} e_{k, i}^{m}\right]^{T} \\
& =\Phi_{m}(x)^{T}\left[e_{k, 0}, e_{k, 1}, \ldots, e_{k, m}\right] c=\Phi_{m}(x)^{T} V_{k} c
\end{aligned}
$$

where $V_{k}(k=0,1, \ldots m)$ is an $(m+1) \times(m+1)$ matrix that has vectors $e_{k, i}(i=0,1, \ldots, m)$ for each column's. If we define $\bar{C}=\left[V_{0} c, V_{1} c, \ldots, V_{m} c\right]$, then we get 
$c^{T} \Phi_{m}(x) \Phi_{m}(x)^{T} \approx \Phi_{m}(x)^{T} \bar{C} A^{T}$

and therefore we obtain the operational matrix of product $\hat{C}=\bar{C} A^{T}$.

Corollary 3.6. Suppose $y(t) \approx c^{T} \Phi_{m}(t), \quad x(t) \approx d^{T} \Phi_{m}(t)$ and $\hat{C}_{(m+1) \times(m+1)}$ be the operational matrix of product using BPs for vector $c$. We can get the approximate function for $x(t) y(t)$ using BPs as follows:

$$
y(t) x(t) \approx \Phi_{m}(t)^{T} \hat{C} d .
$$

Proof. By using Lemma 3.5, it is clear.

Corollary 3.7. Suppose $y(t) \approx c^{T} \Phi_{m}(t)$ and $\hat{C}_{(m+1) \times(m+1)}$ be the operational matrix of product using BPs for vector $c$. We can get the approximate function for $y^{k}(t) \quad(k \in N)$, using BPs as follows:

$y^{k}(t) \approx \Phi_{m}(t)^{T} \tilde{C}_{k}$,

where $\tilde{C}_{k}=\hat{C}^{k-1} c$.

Proof. By using induction, we can obtain approximation for $y^{k}(t),(k \in \mathrm{N})$ as follows:

For $k=1$ by (12) we have $y(t) \approx c^{T} \Phi_{m}(t)$. Also, for $k=2$ by Lemma 3.5 we obtain

$y^{2}(t) \approx c^{T} \Phi_{m}(t) \Phi_{m}(t)^{T} c \approx \Phi_{m}(t)^{T} \hat{C} c$.

Then, for $k=3$ we get $y^{3}(t) \approx c^{T} \Phi_{m}(t) \Phi_{m}(t)^{T} \hat{C} c \approx \Phi_{m}(t)^{T} \hat{C}^{2} c$.

So, by induction we can write

$y^{k}(t) \approx c^{T} \Phi_{m}(t) \Phi_{m}(t)^{T} \hat{C}^{k-2} c \approx \Phi_{m}(t)^{T} \tilde{C}_{k}$,

where $\widetilde{C}_{k}=\hat{C}^{k-1} c$

\section{BPs operational matrix for fractional derivative}

In this section, we obtain the operational matrix for the fractional derivative. We can write $D^{\alpha} \Phi_{m}(t)=\frac{1}{\Gamma(n-\alpha)} t^{n-\alpha-1} * \Phi_{m}^{(n)}(t), \quad 0 \leq t \leq 1$,

where $*$ denotes the convolution product.

By (11) we obtain 
$D^{\alpha} \Phi_{m}(t)=\frac{1}{\Gamma(n-\alpha)} A\left(t^{n-\alpha-1} * T_{m}^{(n)}(t)\right)=A D^{\alpha} T_{m}(t)=A\left[D^{\alpha} 1, D^{\alpha} t, \ldots, D^{\alpha} t^{m}\right]^{T}$,

where $\quad D^{\alpha} t^{j}= \begin{cases}0 & j=0, \ldots,\lceil\alpha\rceil-1, \\ \frac{\Gamma(j+1)}{\Gamma(j+1-\alpha)} t^{j-\alpha} & j=\lceil\alpha\rceil, \ldots, m .\end{cases}$

Therefore we have

$D^{\alpha} T_{m}(t)=\tilde{\Sigma} \tilde{T}$

where $\tilde{\Sigma}$ and $\tilde{T}$ are a $(m+1) \times(m+1)$ diagonal matrix and a $(m+1) \times 1$ matrix, respectively as follows:

$$
\tilde{\Sigma}=\left(\tilde{\Sigma}_{i, j}\right)_{i, j=1}^{m+1}, \quad \tilde{\Sigma}_{i+1, j+1}= \begin{cases}\frac{\Gamma(j+1)}{\Gamma(j+1-\alpha)} & i, j=\lceil\alpha\rceil, \ldots, m \text { and } i=j, \\ 0 & \text { otherwise, }\end{cases}
$$

and $\tilde{T}=(\tilde{T})_{i}=\left\{\begin{array}{ll}0 & i=0, \ldots,\lceil\alpha\rceil-1 \\ t^{i-\alpha} & i=\lceil\alpha\rceil, \ldots, m .\end{array}\right.$.

Now, we approximate $t^{i-\alpha}(i=\lceil\alpha\rceil, \ldots, m)$ with respect to BPs by using (12). Therefore, we can write

$t^{i-\alpha} \approx P_{i}^{T} \Phi_{m}(t)$

where $P_{i},(i=\lceil\alpha\rceil, \ldots, m)$ is a vector $(m+1) \times 1$. So, we have

$$
\begin{aligned}
P_{i}=Q^{-1}\left(\int_{0}^{1} t^{i-\alpha} \Phi_{m}(t) d t\right) & =Q^{-1}\left[\int_{0}^{1} t^{i-\alpha} B_{0, m}(t) d t, \int_{0}^{1} t^{i-\alpha} B_{1, m}(t) d t, \ldots, \int_{0}^{1} t^{i-\alpha} B_{m, m}(t) d t\right]^{T} \\
& =Q^{-1} \bar{P}_{i},
\end{aligned}
$$

where

$$
\begin{aligned}
& \bar{P}_{i}=\left[\bar{P}_{i, 0}, \bar{P}_{i, 1}, \ldots, \bar{P}_{i, m}\right]^{T}, \\
& \bar{P}_{i, j}=\int_{0}^{1} t^{i-\alpha} B_{j, m}(t) d t=\frac{m ! \Gamma(i+j-\alpha+1)}{j ! \Gamma(i+m-\alpha+2)}, \quad i=\lceil\alpha\rceil, \ldots, m \text { and } j=0,1, \ldots, m .
\end{aligned}
$$

Now, we suppose $P$ is an $(m+1) \times(m+1)$ matrix that has vector zero in $\lceil\alpha\rceil$ first column and vector $P_{i}$ in (i+1)th column's for $i=\lceil\alpha\rceil, \ldots, m$.

Finally, from (18)-(25), we obtain

$$
D^{\alpha} \Phi_{m}(t) \approx D_{\alpha} \Phi_{m}(t)
$$

where 
$D_{\alpha} \approx A \tilde{\Sigma} P^{T}$,

is called the Bernstein polynomials operational matrix of fractional derivative.

\section{BPs for solving nonlinear fractional differential equations}

Using Lemma 3.3, we can approximate the functions $x(t)$ as follows:

$y(t) \approx C^{T} \Phi_{m}(t)$,

where $C \in R^{m+1}$.

by (28) and (26) we can write

$D^{\alpha} y(t) \approx C^{T} D_{\alpha} \Phi_{m}(t)$.

Therefore, the problem (1) and (2) reduce to the following problem:

$C^{T} D_{\alpha} \Phi_{m}(t)=g\left(t, C^{T} \Phi_{m}(t)\right)$,

and the initial condition

$C^{T} D_{i} \Phi_{m}(0)=y_{0}^{i}, i=0,1, \ldots,\lceil\alpha\rceil-1$

Now, using Lemma 3.3 we can approximate all of the known functions in (30). Then, by using Lemma 3.5, Corollaries 3.6 and 3.7, since function $g$ is polynomial, we obtain the following approximations:

$g(t, y(t)) \approx G(C) \Phi_{m}(t)$,

where $G: R^{(m+1) \times 1} \rightarrow R^{1 \times(m+1)}$.

Also, by using tau method [20] we can generate algebraic equations from (30) and (32) as follows

$\tilde{G}_{j}=\int_{0}^{1}\left(C^{T} D_{\alpha}-G(C)\right) \Phi_{m}(t) B_{j, m}(t) d t=0, \quad j=0, \ldots, m-\lceil\alpha\rceil$

and from (31) we set $\widetilde{G}_{m-\lceil\alpha\rceil+i+1}=C^{T} D_{i} \Phi_{m}(0)-y_{0}^{i}$ for $i=0,1, \ldots,\lceil\alpha\rceil-1$.

Finally, the problem (1) and (2) has been reduced to the system of algebraic equations

$\tilde{G}_{j}(C)=0, \quad j=0, \ldots, m$.

The above system can be solved for $C$ by Newton's iterative method. Then, we get the approximate value of the functions $y(t)$ from (28).

\section{Numerical examples}


To demonstrate the application of the proposed method and its performance, the obtained results for some examples are presented in this section. We define $y_{m}(t)$ and $y(t)$ for the approximate solution and the exact solution, respectively. These examples are considered because either exact solutions are available for them, or they have also been solved using other numerical schemes, by other authors.

Example 1. Consider the following fractional differential equation

$$
D^{1.5} y(t)=t^{1.5} y(t)+4 \sqrt{\frac{t}{\pi}}-t^{3.5}, \quad 0<t \leq 1
$$

with the following initial conditions

$$
y(0)=0, \quad y^{\prime}(0)=0 .
$$

We know that the exact solution is $y(t)=t^{2}$. The obtained results of BPs for $m=10$ are reported in Table 1 and are plotted in Fig 1 . We observe that our method is very effective.

\begin{tabular}{|c|c|c|c|c|c|}
\hline$t$ & 0.1 & 0.3 & 0.5 & 0.7 & 9 \\
\hline Absolute error & $3.62030 \times 10^{-11}$ & $3.40588 \times 10^{-9}$ & $8.04365 \times 10^{-9}$ & $1.32341 \times 10^{-8}$ & $1.61286 \times 10^{-8}$ \\
\hline
\end{tabular}

Table 1. Absolute error for different $t$ in example 1.

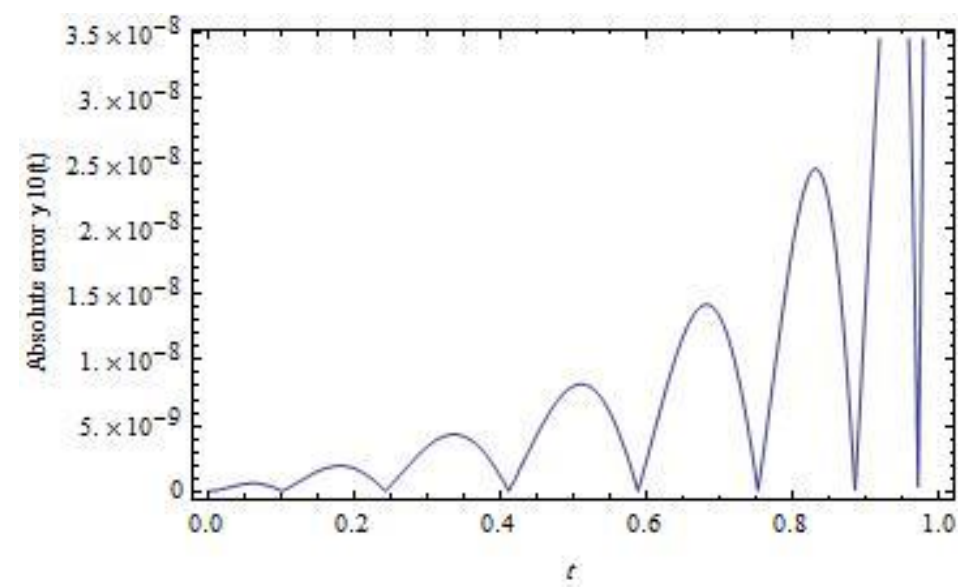

Fig 1. Plot of absolute error function for $m=10$ in example 1.

Example 2. Consider the nonlinear fractional differential equation [21]

$$
D^{\alpha} y(t)=-y(t)^{2}+1, \quad 0<t \leq 1,
$$

subject to the initial condition as 
$y(0)=0$.

The exact solution of the equation for $\alpha=1$ is given as

$$
y(t)=\frac{e^{2 t}-1}{e^{2 t}+1}
$$

Numerical results compared to Ref. [21] are given in Table 2 and also, Fig 2 show the absolute error for our method for $\alpha=1$ and Fig 3 show behavior $y_{10}(t)$ for different $\alpha$.

Table 2. Numerical results for $\alpha=1$ and $m=10$ in example 2 with comparison to exact solution and Ref. [21]

$\begin{array}{cccc}t & \text { Exact } & \begin{array}{c}\text { Present method } \\ m=10\end{array} & \text { Ref.[21] } \\ & & 0.0996679945194735 & 0.099668 \\ 0.1 & 0.09966799462495585 & 0.19737532033276264 & 0.197375 \\ 0.2 & 0.197375320224904 & 0.2913126132161784 & 0.291313 \\ 0.3 & 0.2913126124515909 & 0.3799489605488069 & 0.379944 \\ 0.4 & 0.37994896225522495 & 0.4621171587819068 & 0.462078 \\ 0.5 & 0.4621171572600097 & 0.537049567016221 & 0.536857 \\ 0.6 & 0.5370495669980353 & 0.6640367728588771 & 0.661706 \\ 0.7 & 0.6043677771171635 & 0.7162978670585518 & 0.709919 \\ 0.8 & 0.664036770267849 & 0.7615941454790327 & 0.746032 \\ 0.9 & 0.7162978701990244 & & \end{array}$

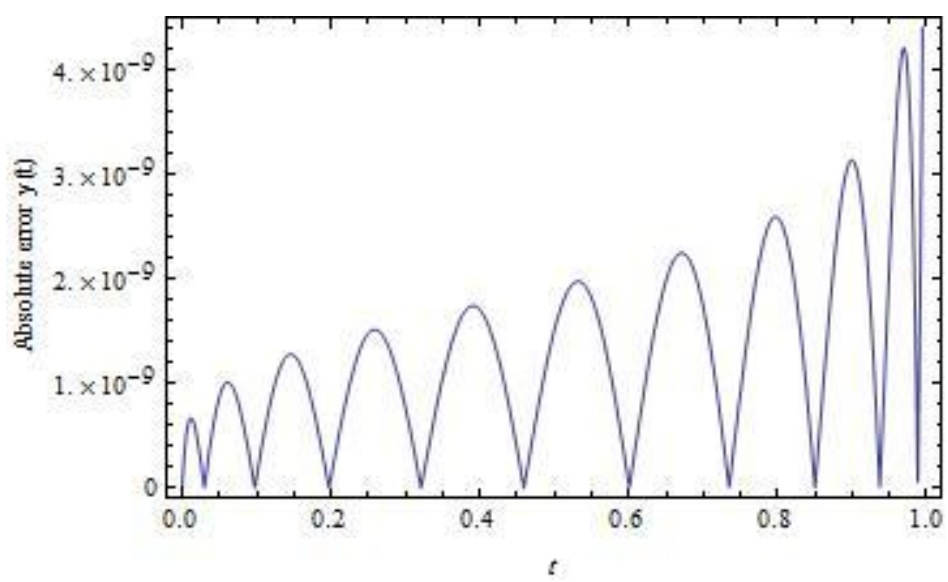

Fig 2. Plot of absolute error function for $\alpha=1$ and $m=10$ in example 2 . 
Mohsen Alipour, Davood Rostamy / TJMCS Vol .5 No.3 (2012) 185-196

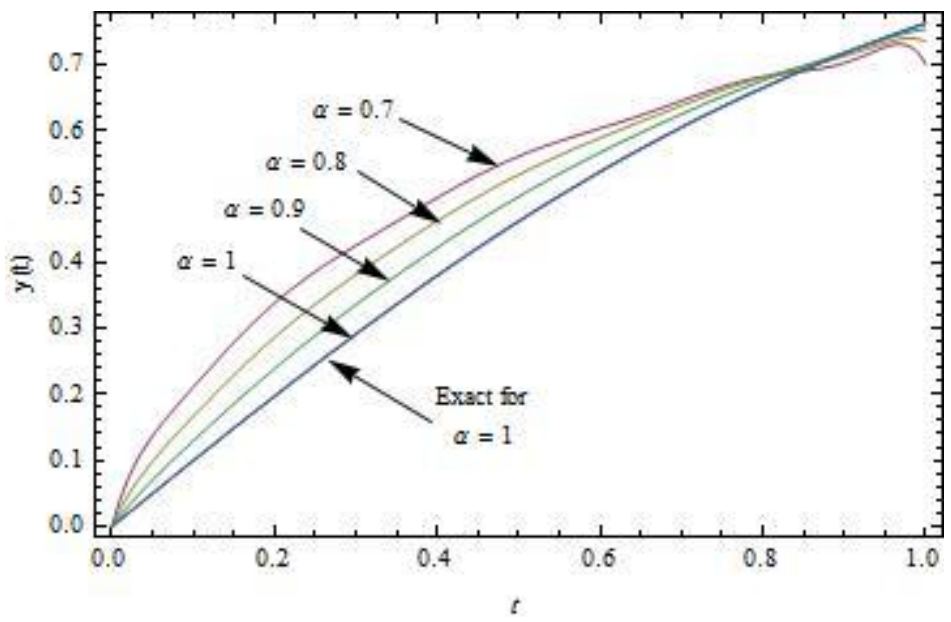

Fig 3. Plot of $y_{10}(t)$ for different $\alpha$ and exact solution for $\alpha=1$ in example 2 .

Example 3. Consider the differential equation of fractional order [21]

$D^{\alpha} y(t)=2 y(t)-y(t)^{2}+1, \quad 0<t \leq 1$,

subject to the initial condition as

$y(0)=0$.

The exact solution of the equation for $\alpha=1$ is given as

$y(t)=1+\sqrt{2} \tanh \left(\sqrt{2} t+\frac{1}{2} \log \left(\frac{\sqrt{2}-1}{\sqrt{2}+1}\right)\right)$.

Numerical results compared to Ref. [21] are given in Table 3 and also, Fig 4 show the absolute error for our method for $\alpha=1$ and Fig 5 show behavior $y_{10}(t)$ for different $\alpha$.

Table 3. Numerical results for $\alpha=1$ and $m=10$ in example 3 with comparison to exact solution and Ref. [21]

$\begin{array}{cccc}t & \text { Exact } & \text { Present method } & \text { Ref.[21] } \\ & & m=10 & \\ 0.1 & 0.11029519691696243 & 0.11029518739630002 & 0.110294 \\ 0.2 & 0.2419767996211093 & 0.24197681569579768 & 0.241965 \\ 0.3 & 0.39510484866037854 & 0.3951048273012639 & 0.395106 \\ 0.4 & 0.567812166292939 & 0.5678121822516022 & 0.568115 \\ 0.5 & 0.756014393431376 & 0.7560144002158702 & 0.757564 \\ 0.6 & 0.953566216471923 & 0.9535661806115561 & 0.958259 \\ 0.7 & 1.1529489669796236 & 1.1529490195918712 & 1.163459 \\ 0.8 & 1.3463636553683758 & 1.346363598686117 & 1.365240 \\ 0.9 & 1.5269113132806247 & 1.5269113915752475 & 1.554960 \\ 1 & 1.6894983915943833 & 1.6894986909366667 & 1.723810\end{array}$




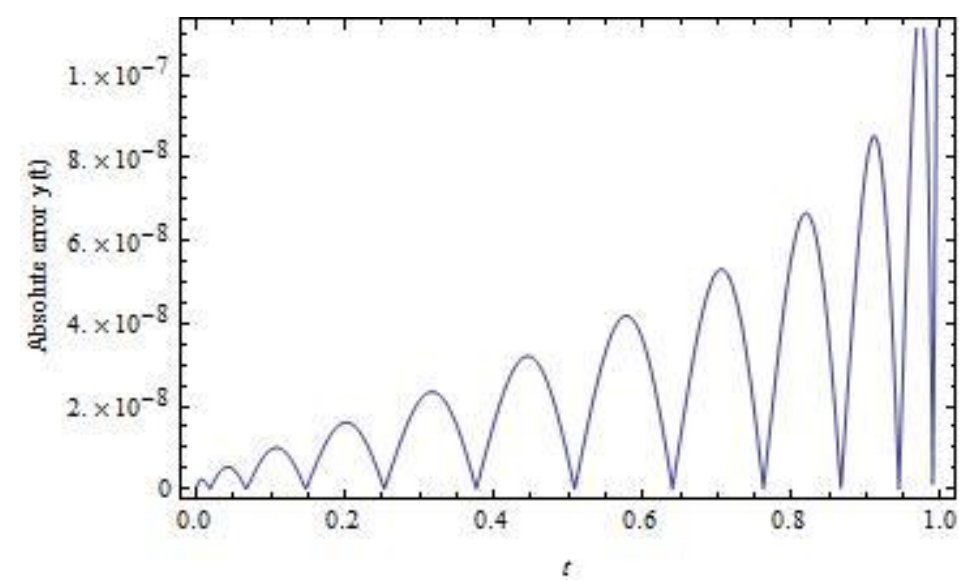

Fig 4. Plot of absolute error function for $\alpha=1$ and $m=10$ in example 3.

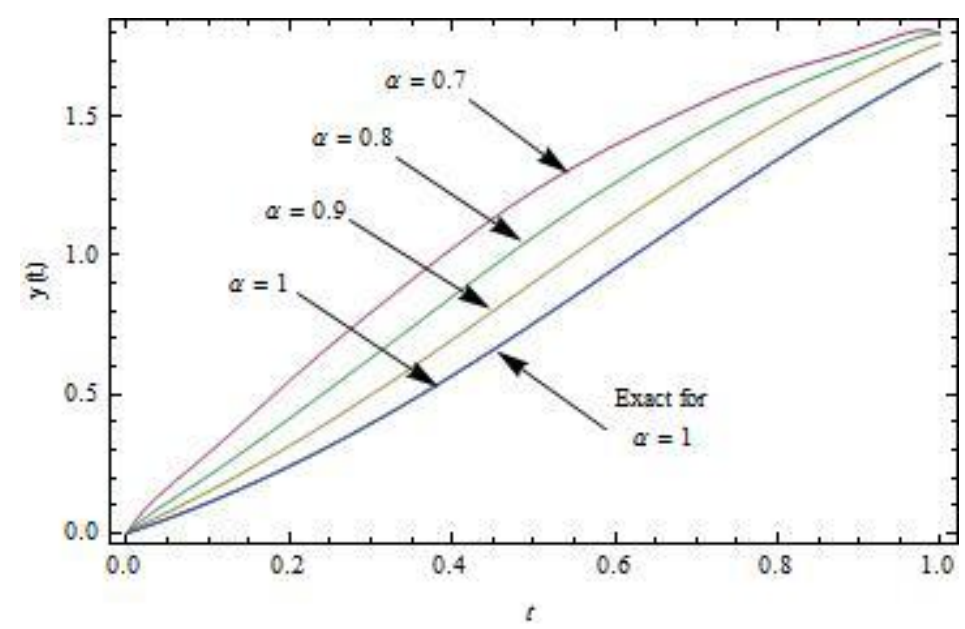

Fig 5. Plot of $y_{10}(t)$ for different $\alpha$ and exact solution for $\alpha=1$ in example 3.

\section{Conclusion}

In this work, we get operational matrices of the product, power and fractional derivative by Bernstein polynomials. Then by using these matrices, we reduced the nonlinear fractional differential equations to a system of algebraic equations that can be solved easily. Finally, numerical examples are simulated to demonstrate the high performance of proposed method. We observed that the obtained results were in good agreement with the exact solution. Also, we saw that the solutions approach to the solutions for differential equation with derivative order 1 , as the order of the fractional derivative approaches to 1 .

\section{References}

[1] X. Gao, J.Yu, Synchronization of two coupled fractional-order chaotic oscillators, Chaos Sol. Fract. 26 (1) (2005) 141-145.

[2] J.G. Lu, Chaotic dynamics and synchronization of fractional-order Arneodo's systems, Chaos Sol. Fract. 26 (4) (2005) 1125-1133.

[3] J.G. Lu, G. Chen, A note on the fractional-order Chen system, Chaos Sol. Fract. 27 (3) (2006) 685-688.

[4] I. Podlubny, Fractional Differential Equations, Academic Press, NewYork, 1999.

[5] S. Momani, Z. Odibat, Homotopy perturbation method for nonlinear partial differential equations of fractional order, Phys. Lett. A 365 (5-6) (2007) 345-350. 
[6] S. Momani, Z. Odibat, Numerical comparison of methods for solving linear differential equations of fractional order, Chaos Soliton. Fract. 31 (5) (2007) 1248-1255.

[7] Z. Odibat, S. Momani, Modified homotopy perturbation method: application to quadratic Riccati differential equation of fractional order, Chaos Soliton. Fract. 36 (1) (2008) 167-174.

[8] M. Zurigat, S. Momani, Z. Odibat, A. Alawneh, The homotopy analysis method for handling systems of fractional differential equations, Applied Mathematical Modelling 34 (2010) 24-35.

[9] S. Momani, Al-Khaled, Numerical solutions for systems of fractional differential equations by the decomposition method, Appl. Math. Comput. 162 (3) (2005) 1351-1365.

[10] H. Jafari, V.D. Gejji, Solving a system of nonlinear fractional differential equations using Adomain decomposition, Appl. Math. Comput. 196 (2006) 644-651.

[11] V. Daftardar-Gejji, H. Jafari, Adomian decomposition: a tool for solving a system of fractional differential equations, J. Math. Anal. Appl. 301 (2) (2005) 508-518.

[12] Z. Odibat, S. Momani, Application of variational iteration method to nonlinear differential equations of fractional order, Int. J. Nonlinear Sci. Numer. Simulat. 1 (7) (2006) 15-27.

[13] V. Daftardar-Gejji, H. Jafari, An iterative method for solving nonlinear functional equations, J. Math. Anal. Appl. 316 (2006) 753-763.

[14] V. S. Ertürk, S. Momani, Solving systems of fractional differential equations using differential transform method, Journal of Computational and Applied Mathematics 215 (2008) 142 - 151.

[15] M. Caputo, Linear models of dissipation whose $Q$ is almost frequency independent II, Geophys. J. Roy. Astronom. Soc. 13 (1967) 529-539.

[16] Y. Luchko and R. Gorneflo, The initial value problem for some fractional differential equations with the Caputo derivative, Preprint series A08-98, Fachbereich Mathematik und Informatik, Freie Universitat Berlin, 1998.

[17] K. S. Miller, B. Ross, An Introduction to the Fractional Calculus and Fractional Differential Equations, John Wiley and Sons, Inc., New York, 1993.

[18] K.B. Oldham and J. Spanier, The Fractional Calculus, Academic Press, New York, 1974.

[19] E. Kreyszig, Introduction Functional Analysis with Applications, John Wiley and Sons Incorporated, 1978.

[20] C. Canuto, M.Y. Hussaini, A. Quarteroni, T.A. Zang, Spectral Methods in Fluid Dynamic, Prentice-Hall, Englewood Cliffs, NJ, 1988.

[21] Z. Odibat,S. Momani, Modified homotopy perturbation method: Application to quadratic Riccati differential equation of fractional order, Chaos. Solitons and Fractals. 36 (2008) 167-174. 